\title{
BMJ Open Patient safety priorities in mental healthcare in Switzerland: a modified Delphi study
}

\author{
Anna C Mascherek, ${ }^{1}$ David L B Schwappach ${ }^{1,2}$
}

To cite: Mascherek AC, Schwappach DLB. Patient safety priorities in mental healthcare in Switzerland: a modified Delphi study. BMJ Open 2016;6:e011494. doi:10.1136/bmjopen-2016011494

- Prepublication history for this paper is available online. To view these files please visit the journal online (http://dx.doi.org/10.1136/ bmjopen-2016-011494).

Received 15 February 2016 Revised 1 June 2016 Accepted 18 July 2016
CrossMark

\footnotetext{
${ }^{1}$ Patient Safety Switzerland, Zürich, Switzerland ${ }^{2}$ Institute of Social and Preventive Medicine (ISPM), University of Bern, Bern, Switzerland
}

Correspondence to Dr David L B Schwappach; schwappach@ patientensicherheit.ch

\section{ABSTRACT}

Objective: Identifying patient safety priorities in mental healthcare is an emerging issue. A variety of aspects of patient safety in medical care apply for patient safety in mental care as well. However, specific aspects may be different as a consequence of special characteristics of patients, setting and treatment. The aim of the present study was to combine knowledge from the field and research and bundle existing initiatives and projects to define patient safety priorities in mental healthcare in Switzerland. The present study draws on national expert panels, namely, round-table discussion and modified Delphi consensus method.

Design: As preparation for the modified Delphi questionnaire, two round-table discussions and one semistructured questionnaire were conducted. Preparative work was conducted between May 2015 and October 2015. The modified Delphi was conducted to gauge experts' opinion on priorities in patient safety in mental healthcare in Switzerland. In two independent rating rounds, experts made private ratings. The modified Delphi was conducted in winter 2015.

Results: Nine topics were defined along the treatment pathway: diagnostic errors, non-drug treatment errors, medication errors, errors related to coercive measures, errors related to aggression management against self and others, errors in treatment of suicidal patients, communication errors, errors at interfaces of care and structural errors.

Conclusions: Patient safety is considered as an important topic of quality in mental healthcare among experts, but it has been seriously neglected up until now. Activities in research and in practice are needed. Structural errors and diagnostics were given highest priority. From the topics identified, some are overlapping with important aspects of patient safety in medical care; however, some core aspects are unique.

\section{BACKGROUND}

Identifying patient safety priorities in mental healthcare is an emerging issue. Mental healthcare as such has developed into one central field in healthcare management. The incidence rates as well as the economic burden due to mental health conditions

\section{Strengths and limitations of this study}

- Identification of central topics of patient safety in mental healthcare in Switzerland.

- Prioritisation of topics by reference to four dimensions.

- The number of experts was limited.

- Basically no variation emerged in the evaluation of topics on the dimensions 'potential for harm', 'preventability' and 'practicability of improvement activities'. For these dimensions, an error of central tendency cannot be ruled out.

increase. $^{1-6}$ In 2015, the Roadmap for Mental Health Research in Europe (ROAMER) consortium published a paper on mental health research priorities in Europe, emphasising the need of joint action to improve treatment in mental health. ${ }^{7}$ They formulated six priorities in mental health research and well-being research, among which the reduction of stigma and the empowerment of service users and carers were mentioned. Aspects of patient safety are an established area of research and implementation programmes. ${ }^{8}$ However, the focus mainly lies on patient safety in acute medical care and efforts concerning patient safety in mental health remain largely underrepresented. ${ }^{9}$ In 2011, Brickell and McLean ${ }^{9}$ pointed to the general necessity of research on patient safety in mental healthcare to raise awareness and to generate knowledge. A variety of aspects of patient safety in medical care apply for patient safety in mental care as well. Adverse drug events, errors at interfaces of care and communication errors are common in both areas. ${ }^{9-11}$ Besides aspects of patient safety that overlap, several core aspects are unique, making patient safety in mental healthcare a topic of its own. This is the case for the care of suicidal patients, issues of physical restraint and seclusion. The National Health Service (NHS) added suicide during treatment as a never event to their never event list. ${ }^{12}$ 
However, in a study by Gillies et $a l,{ }^{13}$ errors in care-related factors, especially communication and medication, were identified as one substantial aspect contributing to committing suicide in suicidal patients. Other studies corroborate the fact that inpatient care and staff-related factors pose a risk of suicide on suicidal patients. ${ }^{14} 15$ Stigmatisation preventing adequate treatment represents another aspect specific of patient safety in mental healthcare. Stigmatisation has been identified as one important barrier for individuals to seek treatment. ${ }^{16}{ }^{17}$ This has also been acknowledged by the ROAMER recommendations, which endorse investment of resources into research dealing with the reduction of stigmatisation in mental healthcare. ${ }^{7}$ Also, insufficient treatment facilities pose a threat for adequate treatment. Recent data from the Commonwealth Fund show that in Switzerland, only $26 \%$ of general practitioners feel that their practice is well prepared to manage the care of patients with severe mental health issues. ${ }^{18-20}$ Although Briner and Manser $^{21}$ published a study on main clinical risk management topics in mental healthcare in Switzerland in 2013, the study falls short on explicitly analysing the general need of research and information on patient safety. To the best of our knowledge, as in many other countries no comprehensive study specifically describes aspects of patient safety in mental healthcare in Switzerland. The aim of the present study was therefore to combine knowledge from the field and research and bundle existing initiatives and projects to define patient safety priorities in mental healthcare in Switzerland. The present study draws on national expert panels, namely, round-table discussion and a modified Delphi consensus method. In areas where there is little or no data on a research topic, expert panels have been established as valuable sources of information. ${ }^{20}$ 22-24 Aim of the present study was, first, to define relevant topics of patient safety in mental healthcare and, second, to prioritise these topics according to expert ratings. The present study was organised as two consecutive substudies. In the first study, a round-table discussion and a semistructured survey were conducted as preparation to define relevant topics. In the second study, a modified Delphi study was conducted to define patient safety priorities in mental healthcare in Switzerland.

\section{METHOD/STUDY DESIGN}

\section{Preparation: round-table discussion and survey}

As preparation and prerequisite of the development of the modified Delphi questionnaire, two round-table discussions and one semistructured questionnaire were conducted. The first round-table discussion served as a starting point. Information was then used to conduct the semistructured questionnaire. Results from the questionnaire, then again, were used as basis for discussion at the second round-table discussion. The first round-table discussion took place in May 2015, the second in
October 2015. The survey was conducted in August 2015. See figure 1 for an illustration of the study design. Participation at the round table and the modified Delphi study as well as completion of the questionnaire were regarded as informed consent. Study design and data collection did not require approval of an ethical committee in Switzerland referring to Articles 1 and 2 of the Federal Act on Research involving Human Beings (Human Research Act, HRA). ${ }^{25}$

\section{Round table}

\section{Round-table participants}

For the round-table discussions, 18 participants were invited. All participants were recruited independently and on the basis of their expertise. Before sampling, we generated a grid in order to cover all relevant specialties, professions and settings. Following purposive sampling, individuals were recruited in order to cover all aspects with a reasonable number of individuals. Relevant professions were psychologists, physicians, nursing staff, psychotherapists, general practitioner, patients, and scientists. Experts were supposed to represent outpatient as well as inpatient care. Child and adolescent medicine, geriatrics, psychiatry and psychology were considered relevant specialties. After establishing this grid, experts were recruited with purposive sampling. A total of 13 experts participated at the first round-table meeting

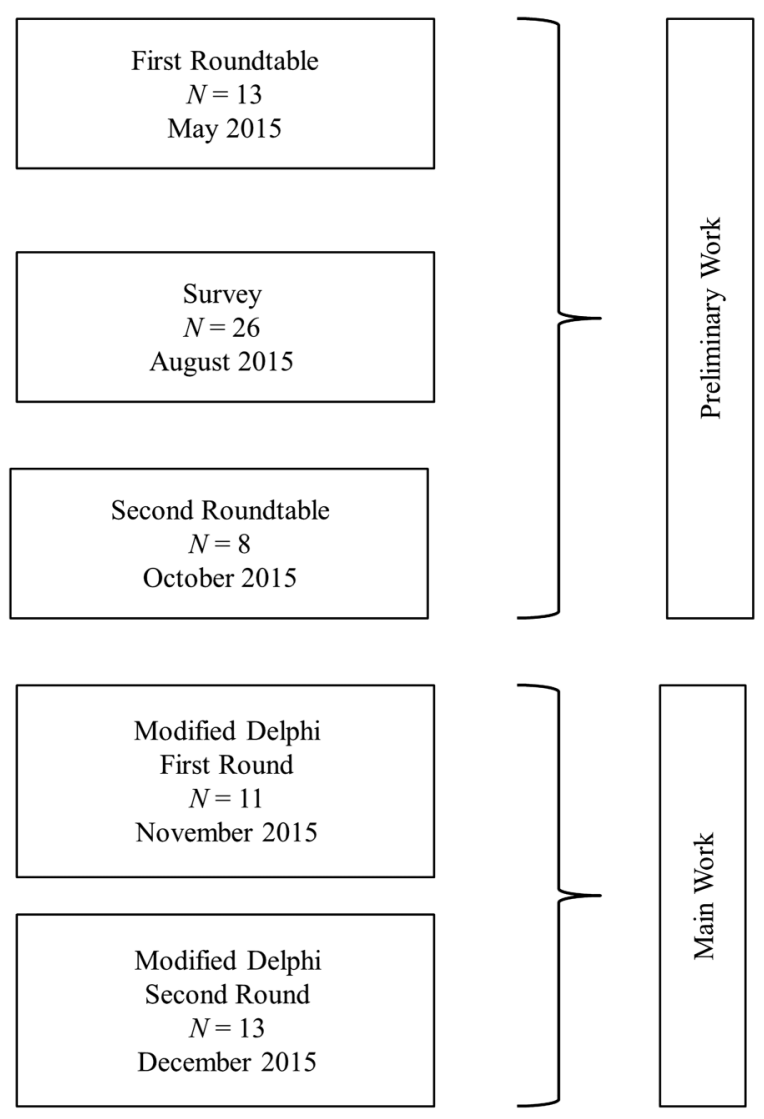

Figure 1 Illustration of study design. 
(7 females, 6 males), 8 participated at the second meeting (6 females, 2 males). Participants covered a broad range of clinical and research areas as well as different professions including nurses $(n=3)$, medical doctors $(n=2)$, psychiatrists $(n=4)$, risk managers $(n=2)$ and psychotherapists $(n=2)$. Participants worked in research $(n=2)$, outpatient care $(n=4)$ and inpatient care $(n=6)$, in large facilities or small community services, as consultative support or as general practitioner. Two patient advocates had expertise across a variety of settings and were members of two different patient organisations. General mental health patients as well as special subpopulations such as geriatrics, addiction medicine, children and youth, and forensics were covered by the workplaces of the experts. All participants held expertise in more than one of the areas covered; hence, numbers in brackets do not add up to sample size. All experts predominantly were experts for their specialty, however, also had some experience in quality management and some even in patient safety.

\section{Round-table discussion}

For the round table, participants were informed in advance about main topics of each meeting and supplemental material for preparation was sent. Main topics of the first round-table discussion were the definition of whether and what is unique in patient safety in mental healthcare and the definition of important hotspots in the area of mental healthcare in Switzerland. At the first round table, consensus on a general definition of patient safety in mental healthcare was achieved and distinct hotspots were discussed. At the second meeting, nine topics that were defined by the research team according to the experts' input and as a result of the questionnaire were discussed and refined. The defined topics were the basic categories to be evaluated in the modified Delphi questionnaire and are described in detail below.

\section{Semistructured questionnaire}

To validate the results from the first round-table discussion and gather additional information from the field, a semistructured questionnaire was applied. Participants were recruited via snowball sampling. The questionnaire consisted of three open questions that should be answered in free-text form. The first question was 'Please describe situations within the healthcare setting in which you witnessed or experienced patient safety relevant adverse events'. The second question was 'Please write down the two most important hotspots in patient safety from your point of view' and the third question was 'Do you know of any projects whatsoever that deal with aspects relevant for the improvement of patient safety in mental health?'. In total, 26 questionnaires were returned. Data were grouped together with the information from the first round table and were discussed at the second round-table discussion. Almost no existing projects and activities related to patient safety were reported in the survey. This result points to the need of making activities of patient safety in mental healthcare priority. Nine topics emerged: diagnostic errors, non-drug-related treatment errors, medication errors, errors related to coercive measures, errors in the management of aggression against self and others, errors in treatment of suicidal patients, communications errors, errors at interfaces of care and structural errors. All topics are located along the clinical treatment pathway. Communication errors and errors at interfaces of care refer to errors that occur all along the treatment pathways and are rather unspecific. Structural errors emerge on latent levels, that is, policy levels. Shortcomings in infrastructure necessary for adequate treatment were described as structural errors. Namely, this comprises staff shortage, too few treatment facilities for special subgroups of patients (eg, children), and insufficient subsequent treatment options. All errors that occur within the diagnostic process were defined as diagnostic errors. The remaining six topics relate to specific aspects during treatment. The definition of the topics is presented in table 1 .

\section{Main work: modified Delphi study}

Following the round-table discussion and the survey, the modified Delphi method was applied to gauge experts' opinion on priorities in patient safety in mental healthcare in Switzerland. In two independent rating rounds, experts made private ratings. Between rounds, data were aggregated by the research team to provide anonymous feedback to the experts on their and the other experts' ratings. For both rounds, the modified Delphi questionnaire was sent to the expert panel of the round table, except two who dropped out for different reasons. Eight experts that were not invited to the round tables were additionally recruited for the modified Delphi. Those eight individuals were recruited to add expertise without prior participation at the round-table discussion. Additionally invited experts covered multiple aspects of the grid as well; two were female, six were male. We recruited experts from research $(n=2)$, risk management $(n=2)$, patient advocates $(n=1)$, psychiatrists $(n=2)$, psychotherapists $(n=1)$ and medical doctors $(n=2)$. Experts worked in inpatient care $(n=4)$ and outpatient care $(\mathrm{n}=3)$. In total, the modified Delphi questionnaire was sent to 24 individuals with the invitation to participate. The modified Delphi was conducted between beginning of November 2015 and end of December 2015 with $\sim 2$ weeks' time to provide the ratings.

\section{First round}

The experts' task in the first round was to prioritise the nine defined topics on four dimensions and in one global evaluation. Each of the four dimensions was to be rated on a four-point rating scale. The four dimensions were 'frequency', 'potential for harm', 'preventability' and 'practicability of improvement activities'. 'Frequency' was defined as the frequency with which 
Table 1 Definition and examples of nine topics defined at the round-table discussion

\begin{tabular}{ll}
\hline Topic & Definition \\
\hline Diagnostic errors & Errors in diagnostics. Misdiagnosed and/or \\
& underdiagnosed patients, wrong diagnoses, \\
& errors in differential diagnoses. Insufficient \\
& medical clarification in patients with mental \\
& illnesses and vice versa.
\end{tabular}

Non-drug treatment errors

Medication errors

Errors related to coercive measures

Errors related to aggression management against self and others

\section{Errors in treatment of} suicidal patients

\section{Communication errors}

Errors at interfaces of care

Structural errors
Any error that occurs in non-drug therapeutic interventions. This could be the allocation to a wrong intervention or institution or errors conducted over the course of psychotherapy. Any errors that result in deviations from the ideal medication process, eg, errors of prescription, administration, confusion and unconsidered interactions between drugs. Regular side effects do not classify as medication errors.

Any errors that occur before, during and after the use of coercive measures. Unnecessary use of coercive measure or insufficient information and analysis of measures with patient

Any errors that occur during treatment of aggression against self and others. An error could be failure to prevent aggression against others in inpatients.

Any errors that occur during treatment of suicidal tendencies. This could be insufficient clarification/screening of suicidal tendencies or wrong or insufficient introduction of measures to prevent suicide.

Any errors occurring in any form of communication between professionals and patients and relatives. Communication errors are unspecific and may occur all along the treatment pathway. Errors are insufficient patient information about treatment, alternatives, insufficient patient involvement in decision-making or lack of professional interpreters.

Errors concerning any handovers and transfers of patients between or within institutions This could be lack of information at transfer or incomplete documentation in medical records.

Errors on latent level that lead to active failures at the sharp end. Errors occur as a result of policy decisions, but are actually conducted by practitioners. Possible errors are understaffing, lack of treatment facilities for subgroups of patients (children, older adults)

\section{Example}

Patient is treated for depression by a psychotherapist but does not get better. During a medical consultation hypothyroidism is diagnosed. After medical treatment patient gets better.

A patient aged 8 years gets psychotherapeutic treatment. Her mother, as the closest reference person, is not involved into therapeutic process.

Instead of $5 \mathrm{mg} / \mathrm{mL}$, patient is dosed with $10 \mathrm{mg} / \mathrm{mL}$ of haloperidol.

Patient is restraint. Owing to Valium intake, she does not remember the restraint, but imprints at her wrist tell from the restraint. Staff fails to inform about restraint.

Patient attacks roommate late at night without obvious reason. Patient was aggressive during the day already; however, no actions were taken.

Patient strangles herself as a consequence of her depression. She was in her room without sufficient monitoring.

Patient with psychotic disorder accidentally listens to a conversation between her husband and the attending physician. The doctor says: "This won't be easy. This will take time."

Patient on oncological ward gets also treated for a depressive episode. The episode is not documented in patient chart. As unknown to staff, depression treatment is not continued at the rehabilitation centre. Patient decompensates.

Owing to lack of space, a patient aged 15 years has to stay in the adult ward for crisis intervention. errors and preventable adverse events occur in the respective areas. The rating scale for frequency was 'very frequent', 'frequent', 'occasionally' and 'rarely'. 'Potential for harm' described the potential damage errors and preventable adverse events caused in the respective areas. Answer options here were 'major potential for harm', 'moderate potential for harm', 'minor potential for harm' and 'insignificant potential for harm'. 'Preventability' described as to how clear errors were deemed preventable in the respective topics. Included into this rating was the existence of guidelines. Categories were 'clearly preventable', 'preventable', 
'hardly preventable' and 'not preventable'. 'Practicability of improvement activities' was defined as the extent to which interventions and activities in patient safety would be perceived as helpful or would rather be reacted upon with resistance. Categories were 'clearly practicable', 'practicable', 'less practicable' and 'not practicable'. For the global evaluation, topics had to be rated with respect to their overall importance. The individual rankings of the topics were brought together by the research team. The extent of agreement between experts was colour marked. The cut-off criterion for 'high agreement' was an identical rating by at least 7 out of 11 participants; 'medium agreement' was reached if ratings were equally spread over two adjoined categories or 6 out of 11 participants provided identical ratings. Other patterns of ratings were categorised as 'low agreement'. For the global evaluations, median ranking was applied to establish a general ranking out of the individual private rankings of the experts.

\section{Second round}

In the second round, experts were presented the combined rating prepared by the research team and were asked to indicate the extent to which they could agree. Categories were 'completely agree', 'agree with slight differences' and 'do not agree'. Patient safety topics were grouped on each dimension and on one global evaluation. Hence, experts had to rate five lists, which were 'frequency', 'potential for harm', 'preventability', 'practicability of improvement activities' and 'global evaluation'. In a final step, the individual rankings made by the experts were combined to one final ranking by the research team.

\section{RESULTS}

In the first round, 11 out of 24 invited individuals participated. In the second round, 14 out of 24 invited individuals participated. Hence, response rate was $46 \%$ in the first round and $58 \%$ in the second round.

Ranking of the topics on the four dimensions and the global evaluation reached large agreement between the experts in the first round already. Lowest agreement was reached for the ranking of topics on 'frequency'. Here, three topics (communication, interfaces of care and treatment of suicidal patients) only reached 'low agreement' between experts. For 'potential of harm' only diagnostic errors varied substantially across experts. For 'preventability' low agreement emerged for 'structural' errors. For 'practicability of improvement activities' all ratings reached 'medium agreement' at least. On the basis of attached comments to the ratings, the research team created a ranking of the topics on the four dimensions and the global evaluation as a final ranking. This ranking was sent to the experts with the request for comments or minor changes.

Figure 2 depicts the final classification of topics for 'frequency of errors'. As the ratings of the three other dimensions did not vary between topics, they are not shown in figure 2. Variability between topics emerged for the dimension 'frequency of errors'. The frequency of errors differs between topics according to experts' opinion. For 'potential of harm' errors in all areas were

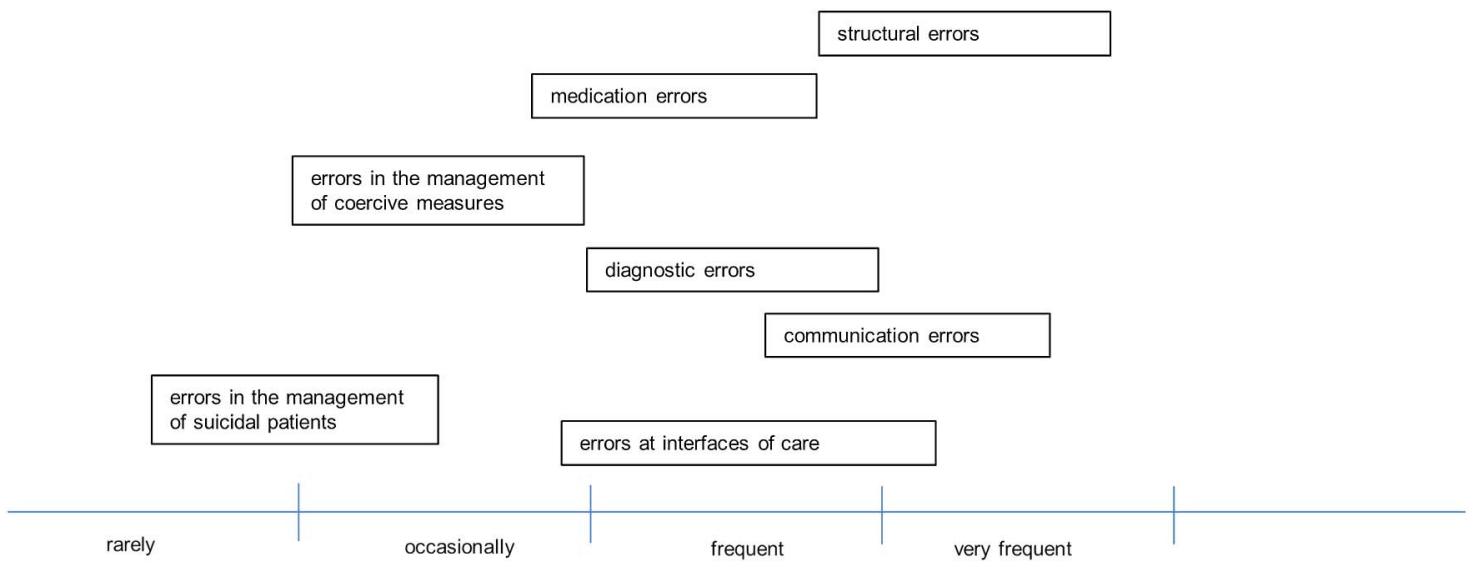

Figure 2 Final experts' ratings of frequency of errors. As almost no variation occurred in the dimension 'preventability', 'potential for harm' and 'practicability of improvement activities', they are not displayed here. 
rated as having the potential of harm. Ratings for 'preventability' and 'practicability of improvement activities' are the same for both dimensions. Experts rate all topics as being preventable and activities as being practicable, except for structural errors. Structural errors are classified as less preventable and less practicable. Concerning the general evaluation, three blocks of different priority emerged (see figure 3). Experts classified the topics 'diagnostic errors' and 'structural errors' as most important. In a second block, experts ranked medication errors, errors in non-drug treatment, errors concerning coercive measures, errors concerning aggression against self and others, and errors in treatment of suicidal patients. In a third and last block, errors at interfaces of care and errors in communication were classified.

\section{DISCUSSION}

In the present study, we set out to identify priorities of patient safety in mental healthcare in Switzerland by drawing on expert rating. We followed two methodological approaches to gather information and prioritise topics. As preparation, two round-table discussions and one survey were conducted to identify key issues relevant to patient safety in mental healthcare. As main work, nine topics were ranked by experts according to priority in a modified Delphi study. Topics can specifically be described and separated. Each error can be systematically analysed and described alongside these dimensions. But although the explicit categorisation is possible, occurring errors will most likely be a combination of different topics. Errors in the management of suicidal patients may, for example, also include insufficient exchange of information. The classification developed in the present study, however, provides a useful framework for describing and analysing patient safety issues in mental healthcare easily understandable. Topics are arranged along the treatment pathway of patients in mental healthcare. Errors in communication and at interfaces of care are 'meta-topics' as they are unspecific and can occur anytime during treatment.

By ranking the nine defined topics in order of importance in a general evaluation, priorities for activities in patient safety in mental healthcare were defined. The present prioritisation goes beyond individual evaluation. Owing to the two rounds in which consensus between experts was achieved the prioritisation reflects the combined evaluation of several experts of the field. Diagnostic errors and structural errors were ranked as most important. Diagnostic errors occur early in treatment. Hence, they are of immense importance and have great impact on the patient and the treatment as following interventions are based on the initial diagnosis. Especially at risk of being misdiagnosed and/or underdiagnosed are patients with comorbidities across disciplines, for example, diagnosing a medical condition in patients who are treated as psychiatric patients and vice versa. ${ }^{26}$ Structural errors represent a different type of errors, however, equally substantial and important. Structural errors emerge on latent level as a consequence of resource allocation by policymakers and
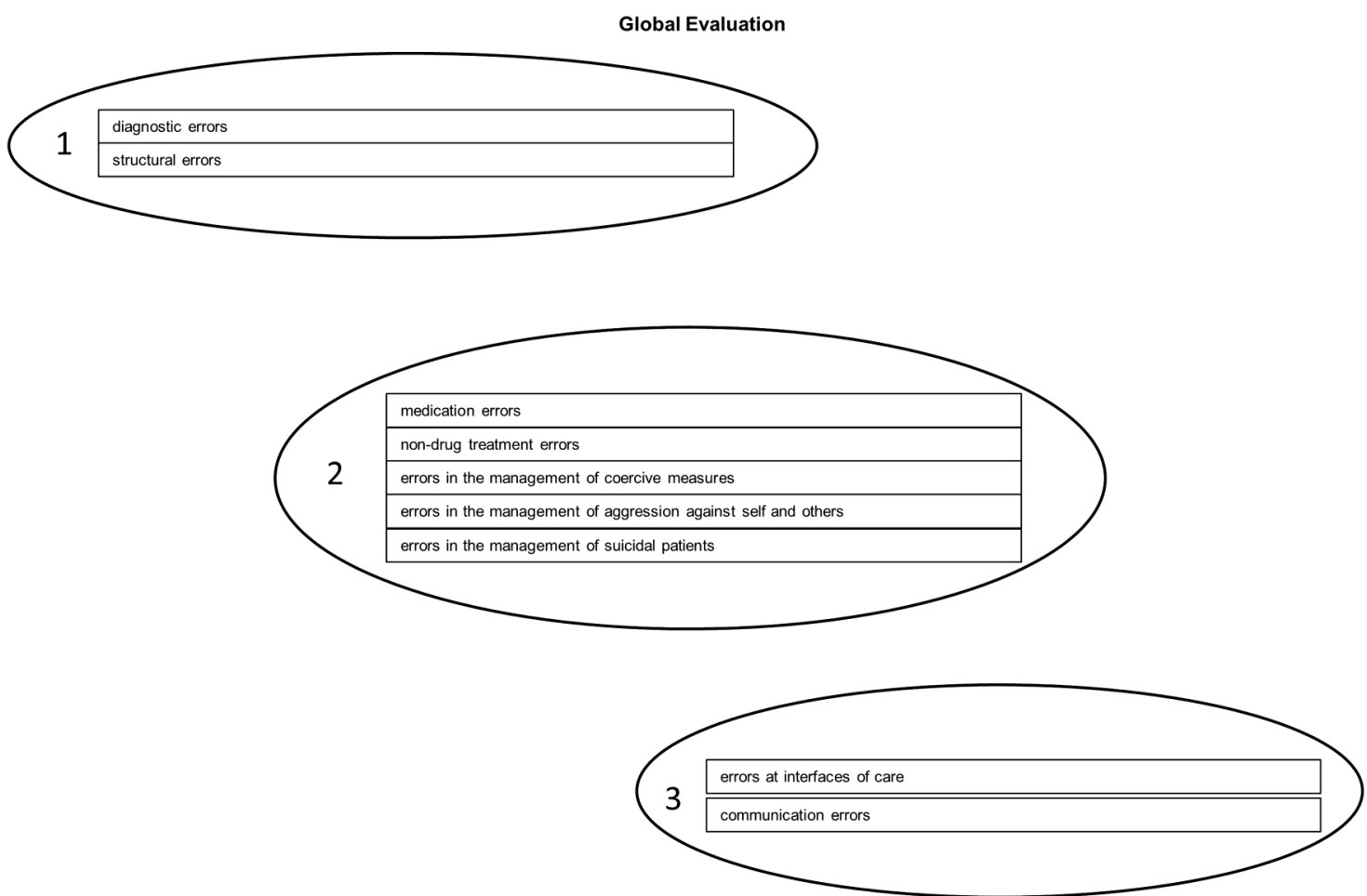

Figure 3 Final experts' priority rating of topics. Topics are grouped into three blocks of different priority. Topics within one block are of equal priority but for different reasons. 
sociopolitical situation. Decisions on political level may result in understaffing or restricted access to sufficient treatment facilities for special subgroups. Staff working at the 'sharp end' is, thus, enforced to commit an error during treatment without being able to prevent it, for example, transferring children to adult wards due to limited treatment places for children. The existing discrepancy between people who initiate an error on latent level and people who are enforced to actually commit the error to the patient against their better knowledge makes structural errors a priority.

The topics classified as second most important are specific aspects of the actual treatment. This is reasonable as the treatment represents the main focus of the total intervention. However, topics were classified for different reasons. Medication errors have received attention already in medical healthcare. Interventions here may be applicable to the context of mental healthcare. Importance may rather come from the fact that although a solid body of interventions as well as research on this topic exists, adverse drug events still pose a serious risk on patients. Hence, it is still necessary to rate medication errors as one important topic of patient safety. Errors in non-drug treatment pose a risk for different reasons. They cover errors during psychotherapy in particular. Dropping out of therapy may be considered as side effect or as treatment error. Harm in mutual trust may also origin in errors conducted by the therapist. Although it is known that such errors occur in non-drug treatment and interventions can cause serious harm, it is still more difficult to define what actually classifies as 'error'; what may be side effects and what can be considered as harm. Although a body of research exists, ${ }^{27-29}$ much more research is needed to shed light on this topic. The topics concerning errors in treatment of suicidal patients and the handling of aggression and coercive measures may be classified as highly important, because of the tremendous impact errors in this field may have on the patient. This makes paying attention to these topics almost mandatory in the realm of patient safety in mental healthcare.

In a third block, errors in communication and errors at interfaces of care were classified. Both topics refer to errors that can occur all along the treatment pathway. Errors here may often occur without severe consequences or may mainly result in 'near misses'/ close calls'. Additionally, the causal link between miscommunication, errors at interfaces of care and harm is much more difficult to establish. Consequences of these types of errors may most often remain vague and rather ill defined.

This study has several limitations. First, the number of experts was limited. Although experts were carefully chosen and different settings, different professions and patient representatives were considered, generalisability is limited. This is also true for the size of the modified Delphi panel as Jorm ${ }^{23}$ stated that stability of panels is reached at around 20 or more members. These concerns can be overcome, however, as clear saturation was reached in the variety of topics mentioned and broad consensus was reached in the modified Delphi study. Second, we had a relatively high attrition rate in the modified Delphi study. This might be due to the fact that a majority (about thirds) participated already at the round table. It is possible that they grew tired of being interviewed over the course of the project. Also, attrition might be due to organisational reasons. As the Delphi questionnaire was sent per email without a reminder, it is possible that experts simply missed the deadline. Third, snowball sampling was applied in the semistructured interview. This was mainly due to resource and time constraints. Biased results are one possible risk of this sampling method which cannot be ruled out completely for the present study. Fourth, basically no variation emerged in the evaluation of topics on the dimensions 'potential for harm', 'preventability' and 'practicability of improvement activities'. For those dimensions an error of central tendency cannot be ruled out. This raises the question whether the dimensions chosen truly reflect the most relevant aspects needed for prioritisation. This question has to be addressed in future research.

This study has several practical implications. First, we found that among experts patient safety is considered an important topic of quality in mental healthcare, but it has been seriously neglected up until now. Activities in research and in practice are needed. Second, among the topics that have been identified, some are overlapping with important aspects of patient safety in medical care; however, some core aspects are unique. This implies that activities on different levels are needed. For aspects with overlap, interventions and knowledge may be borrowed from medical care. For topics that are specific to mental healthcare, research and development of interventions are needed. Future studies and research are needed to shed further light on specific aspects of patient safety in mental healthcare and on different needs of different patient subgroups in different settings.

Acknowledgements The authors thank Thilo Beck (Arud Zentren für Suchtmedizin), Jürgen Barth (UniversitätsSpital Zürich), Anne Eschen (Integrierte Psychiatrie Winterthur), Sandra Kern (Psychiatrische Dienste Thurgau), Rolf Müller (Peer+), Ursula Schreiter (Praxis für Psychiatrie Rehalp), Thomas Maier (St. Gallischen Kantonale Psychiatrische Dienste), Armin von Gunten (Centre Hospitalier Universitaire Vaudois), Christina Holzer-Pruss, Matthias Jäger, Hadmut Prün (Psychiatrische Universitätsklinik Zürich), , Majbritt Jensen (Privatklinik Meiringen AG), Maria-Pia Mahler

(Hausarzt-Praxis), Barbara Stiffler Scherrer (Kinder- und Jugendpsychiatrischer Dienst des Kantons Zürich), Jasmin Jossen (Pro Mente Sana), Birgit Watzke (Universität Zürich) for their expertise.

Contributors ACM and DLBS designed the study, developed all instruments and designed and monitored data collection. ACM cleaned and analysed the data, drafted and revised the manuscript. She is guarantor. DLBS revised the manuscript. ACM and DLBS read and approved the final manuscript.

Funding Parts of the preparation of this article were supported by the Swiss Medical Association (FMH).

Competing interests None declared. 
Provenance and peer review Not commissioned; externally peer reviewed.

Data sharing statement Data are available from the corresponding author upon request.

Open Access This is an Open Access article distributed in accordance with the Creative Commons Attribution Non Commercial (CC BY-NC 4.0) license, which permits others to distribute, remix, adapt, build upon this work noncommercially, and license their derivative works on different terms, provided the original work is properly cited and the use is non-commercial. See: http:// creativecommons.org/licenses/by-nc/4.0/

\section{REFERENCES}

1. Wittchen HU, Jacobi F. Size and burden of mental disorders in Europe-a critical review and appraisal of 27 studies. Eur Neuropsychopharmacol 2005;15:357-76.

2. Wittchen HU, Jacobi F, Rehm J, et al. The size and burden of mental disorders and other disorders of the brain in Europe 2010. Eur Neuropsychopharmacol 2011;21:655-79.

3. Weltgesundheitsorganisation. Psychische Gesundheit: Herausforderungen annehmen, Lösungen schaffen. Dänemark: Weltgesundheitsorganisation, 2006.

4. World Health Organization. The World health report: 2001: mental health: new understanding, new hope. 2001. http://www.who.int/whr/ 2001/en/whr01_en.pdf (accessed 1 Feb 2016).

5. Gustavsson A, Svensson M, Jacobi F, et al. Cost of disorders of the brain in Europe 2010. Eur Neuropsychopharmacol 2011;21:718-79.

6. Bundesamt für Sozialversicherungen. Statistiken zur sozialen Sicherheit. IV-Statistik 2013. Bern: Bundesamt für Sozialversicherungen, 2014.

7. Wykes T, Haro JM, Belli SR, et al. Mental health research priorities for Europe. Lancet Psychiatry 2015;2:1036-42.

8. Baines R, Langelaan M, de Bruijne M, et al. How effective are patient safety initiatives? A retrospective patient record review study of changes to patient safety over time. BMJ Qual Saf 2015;24:561-71.

9. Brickell TA, McLean C. Emerging issuses and challenges for improving patient safety in mental health: a qualitative analysis of expert perspectives. J Pat Saf 2011;7:39-44.

10. Holt S, Schmiedl S, Thürmann PA. Potenziell inadäquate Medikation für ältere Menschen: Die PRISCUS-Liste. Dtsch Arztebl International 2010:107:543-51.

11. Dormann $\mathrm{H}$, Sonst $\mathrm{A}$, Müller $\mathrm{F}$, et al. Adverse drug events in older patients admitted as an emergency-the role of potentially inappropriate medication in elderly people (PRISCUS). Dtsch Arztebl Int 2015;110:213-19.

12. NHS England. The never events list; $2013 / 14$ update. 2013 (cited 4 June 2015). http://www england nhs uk/wp-content/uploads/2013/12/ nev-ev-list-1314-clar pdf; http://www.england.nhs.uk/wp-content/ uploads/2013/12/nev-ev-list-1314-clar.pdf

13. Gillies D, Chicop D, O'Halloran P. Root cause analyses of suicides of mental health clients. Crisis 2015;36:316-24.

14. Jayaram G. Inpatient suicide prevention: promoting a culture and system of safety over 30 years of practice. J Psychiatr Pract 2014;20:392-404.

15. Mills PD, Gallimore BI, Watts BV, et al. Suicide attempts and completions in Veterans Affairs nursing home care units and long-term care facilities: a review of root-cause analysis reports. Int J Geriatr Psychiatry 2016;31:518-25.

16. Hanisch SE, Twomey CD, Szeto AC, et al. The effectiveness of interventions targeting the stigma of mental illness at the workplace: a systematic review. BMC Psychiatry 2016;16:1

17. Corrigan PW, Watson AC, Warpinski AC, et al. Stigmatizing attitudes about mental illness and allocation of resources to mental health services. Community Ment Health J 2004;40:297-307.

18. The Commonwealth Fund. International Health Care System Profiles. 2015 (cited 1 February 2016) http://international. commonwealthfund.org/data/

19. Brickell TA, Nicholls TL, Procyshyn RM, et al. Patient safety in mental health. Edmonton, AB: Canadian Patient Safety Institute and Ontario Hospital Association, 2009.

20. Brickell TA, McLean C. Emerging issuses and challenges for improving patient safety in mental health: a qualitative analysis of expert perspectives. J Patient Saf 2011;7:39-44.

21. Briner M, Manser T. Clinical risk management in mental health: a qualitative study of main risks and related organizational management practices. BMC Health Serv Res 2013;13:44.

22. Fink $A$, Kosecoff J, Chassin M, et al. Consensus methods: characteristics and guidelines for use. Am J Public Health 1984;74:979-83.

23. Jorm AF. Using the Delphi expert consensus method in mental health research. Aust N Z J Psychiatry 2015:49:887-97.

24. Jones J, Hunter D. Consensus methods for medical and health services research. BMJ 1995;311:376-80.

25. The Federal Authorities of the Swiss Confederation. Federal Act on Research involving Human Beings. 2011 (cited 18 January 2016). http://www admin ch/opc/en/classified-compilation/20061313/index html; http://www.admin.ch/opc/en/classified-compilation/20061313/ index.html

26. Panagioti M, Stokes J, Esmail A, et al. Multimorbidity and patient safety incidents in primary care: a systematic review and meta-analysis. PLOS ONE 2015;10:e0135947.

27. Dimidjian S, Hollon SD. How would we know if psychotherapy were harmful? Am Psychol 2010;65:21-33.

28. Barlow DH. Negative effects from psychological treatments: a perspective. Am Psychol 2010;65:13-20.

29. Linden M. How to define, find and classify side effects in psychotherapy: from unwanted events to adverse treatment reactions. Clin Psychol Psychother 2013;20:286-96. 